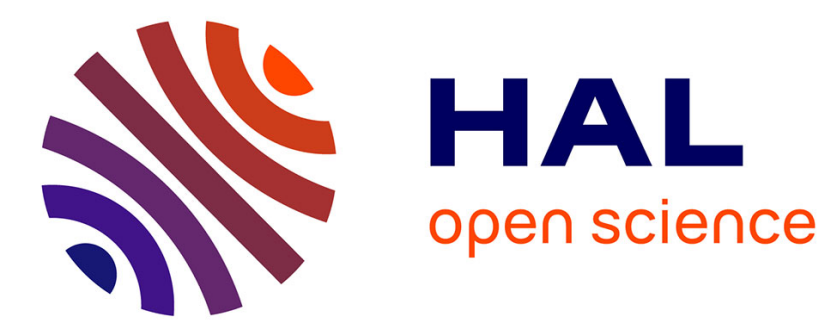

\title{
L'initiative individuelle face à ses obstacles
}

Louis-Marie Barnier

\section{To cite this version:}

Louis-Marie Barnier. L'initiative individuelle face à ses obstacles: Genre et formation professionnelle dans le transport aérien. Cahiers du Genre, 2010, Minoritaires et légitimes, 48, pp.103-125. 10.3917/cdge.048.0103 . hal-03172439

\section{HAL Id: hal-03172439 \\ https://hal.science/hal-03172439}

Submitted on 17 Mar 2021

HAL is a multi-disciplinary open access archive for the deposit and dissemination of scientific research documents, whether they are published or not. The documents may come from teaching and research institutions in France or abroad, or from public or private research centers.
L'archive ouverte pluridisciplinaire HAL, est destinée au dépôt et à la diffusion de documents scientifiques de niveau recherche, publiés ou non, émanant des établissements d'enseignement et de recherche français ou étrangers, des laboratoires publics ou privés. 


\title{
L'initiative individuelle face à ses obstacles
}

\author{
Genre et formation professionnelle \\ dans le transport aérien
}

Louis-Marie Barnier

\begin{abstract}
Résumé
Depuis 2004, la formation professionnelle repose sur l'initiative individuelle du salarié censé être responsable de son parcours. Cette notion de parcours doit être confrontée à la rigidité de certaines situations professionnelles. Dans le transport aérien, les salariés sont assignés à un emploi suivant les normes de genre dans le cadre de la division sociale du travail. L'étude oppose des métiers 'masculins' tel le pilote de ligne, à des métiers tout aussi typés tels l'hôtesse de l'air et de steward, ou l'agent d'enregistrement. Cette étude vise à apporter des éléments pour une discussion critique, au prisme du genre, de l'approche individualisante de la formation professionnelle. ${ }^{1}$
\end{abstract}

TRANSPORT AÉRIEN — FORMATION PROFESSIONNELLE — MÉTIER — INITIATIVE INDIVIDUELLE — COLLECTIF DE TRAVAIL — CONDITIONS DE TRAVAIL

La loi de mai 2004 régissant la formation professionnelle en France repose sur deux logiques complémentaires. D'une part, toute activité salariée est considérée comme un segment au sein

\footnotetext{
${ }^{1}$ Une première version de ce texte a été présentée aux Journées internationales de sociologie du travail, Londres, 2007: «La destinée et le libre arbitre, assignation sociale et formation professionnelle dans le transport aérien ».
} 
d'un parcours professionnel. Le paradigme de la «formation tout au long de la vie » (Clavel, Baunay 2002), mis en avant par la Commission européenne, définit que :

La vie et la poursuite d'une activité professionnelle au sein de la société de la connaissance requièrent des citoyens actifs désireux de mener à bien leur parcours personnel et professionnel ${ }^{2}$.

Dans ce modèle, c'est à travers son parcours professionnel qu'est considérée l'insertion du salarié dans son emploi. Celui-ci doit trouver contrepartie de son engagement à travers un parcours valorisé. Il s'insère alors dans une "logique de parcours continus favorisant des trajectoires personnelles et professionnelles ascendantes ${ }^{3}$. La notion de parcours recouvre ici une acception particulière ${ }^{4}$. Il s'agit d'une succession de situations d'emploi valorisées dont l'individu devrait tirer tous les bénéfices à travers des dispositifs de validation de ces expériences cumulées. Cette notion «dissocie les destins du salarié et de l'entreprise », elle s'inscrit dans une "rhétorique de la 'société du risque' et de la déstabilisation de l'emploi" (Monchatre 2007). L'évolution peut recouvrir différents emplois et périodes d'activités, sous des statuts différents, dans une ou plusieurs entreprises. Loin d'être un simple modèle, cette notion de parcours participe de la configuration du monde social.

D'autre part, l'initiative individuelle est au centre du dispositif de formation. Elle est envisagée comme nouveau mode d'engagement du salarié dans le processus de formation. La " place des choix individuels dans le système » est citée comme nouvelle perspective pour la formation professionnelle par le rapport d'Yves Lichtenberger et Philippe Méhaut (2001), document qui sert de point d'appui pour la négociation de l'accord

\footnotetext{
${ }^{2}$ Mémorandum sur la formation et l'éducation tout au long de la vie, Office des publications officielles des Communautés européennes, Luxembourg, 2000, p 15.

${ }^{3}$ Consultation française sur le mémorandum de la Communauté économique européenne portant sur la formation tout au long de la vie, juillet 2001.

${ }^{4}$ Cette notion se différencie de la mobilité sociale, conçue comme « le trajet d’individus dans l'espace social » (Merllié, Prévot 1991, p. 28) puisque le parcours est envisagé uniquement sous l'angle professionnel, domaine où 'l'individu' peut être valorisé. Elle rejoint davantage la notion de carrière portée par Everett C. Hughes (1937), tout en lui attribuant un sens différent.
} 
interprofessionnel de 2003 portant sur la formation professionnelle. Un Droit individuel à la formation (DIF) ${ }^{5}$ est créé par la loi de 2004 qui succède à l'accord, il autorise les salariés des entreprises privées à demander, à leur initiative, une formation répondant à leur besoin.

Ces deux approches, par le parcours et par l'initiative individuelle, se conjuguent pour construire la figure du salarié censé être maître de son destin professionnel et social. L'individu évoluerait librement, au sein du marché du travail comme au sein de l'entreprise. Il se déplacerait suivant un parcours ascendant, ses initiatives personnelles lui permettant d'effectuer des choix positifs. L'individu, tel que le construit le modèle de la "formation tout au long de la vie ", est un être abstrait, il ne se réfère ni au sexe, ni à la 'race', ni à un quelconque groupe social. Il aurait les mêmes capacités de prendre des initiatives que le citoyen, autre figure abstraite, et souvent mythifiée.

C'est cette figure de l'individu asexué et interclassiste, qui constitue le socle de la construction idéologique de la formation professionnelle, que nous souhaitons interroger ici. Cet individu est censé construire librement son parcours professionnel. L'étude des parcours professionnels dans certains segments du transport aérien ${ }^{6}$, du moins pour la partie intra-entreprise considérée ici, ne valide pas une telle construction de l'individu libre de ses choix, notamment quand l'individu, terme masculin (le neutre n'existant pas en français), est une femme. Deux groupes professionnels particuliers sont majoritairement féminins : les agents de passage qui effectuent l'enregistrement des passagers au moment du départ, et les personnels navigants commerciaux, en charge du service à bord de l'avion. Ils sont considérés à partir de particularités liées au travail, comme exerçant une activité commerciale en relation directe avec le passager. Nous apprécierons les évolutions au sein de ces deux groupes à l'aune d'un

\footnotetext{
${ }^{5}$ Ce droit est de vingt heures de formation par an, cumulables jusqu'à un plafond de cent vingt heures.

${ }^{6}$ Enquête menée dans le cadre d'une thèse (Barnier 2005) et complétée par des données statistiques récentes fournies dans le cadre des instances représentatives du personnel: bilans sociaux, rapports sur la formation professionnelle, etc.
} 
troisième groupe, les pilotes ${ }^{7}$. Ces derniers sont caractérisés par la dimension technique de leur travail ainsi que par le pouvoir que leur confère leur position. La confrontation entre ces deux pôles se fait ici autour de l'usage social inégal de la formation professionnelle. Or les femmes sont présentes dans des emplois moins qualifiés, plus précaires et davantage dans des Petites et moyennes entreprises, elles bénéficient donc moins souvent de la formation que les hommes, mais de plus, dans leur cas, la formation est moins suivie d'effets positifs sur la carrière (Péry 1999). Ce sont ces constats, largement partagés dans le monde de la formation professionnelle, qui ont conduit à l'élaboration de la nouvelle loi. L'initiative individuelle permettrait de réduire les inégalités puisque, dans cette perspective, ce sont les moins formés qui devraient forcément être les plus demandeurs de formation. L'enjeu est donc ici d'analyser, grâce à un éclairage par le genre, l'approche individuelle de la formation professionnelle dans la loi de mai 2004. Quelle place laissent les systèmes organisationnels des entreprises à l'évolution individuelle ? Comment sont utilisés les différents dispositifs à l'initiative du salarié dans le domaine de la formation professionnelle ? Et comment se confrontent ces dispositifs à l'organisation sexuée du travail ${ }^{8}$ dans l'entreprise ?

Après avoir rappelé la façon dont les dispositifs actuels de la formation professionnelle envisagent l'initiative individuelle du salarié, nous verrons comment ces trois segments du transport aérien se structurent autour de la notion de métier et comment ces derniers sont susceptibles (ou non) d'accueillir une démarche d'évolution individuelle.

\footnotetext{
${ }^{7}$ Le groupe des mécaniciens avions au sol, plus proche socialement des agents de passage et des personnels navigants commerciaux (PNC), présente les mêmes caractéristiques du point de vue de l'évolution professionnelle (examens professionnels, possibilité de carrière étendue, et surtout composition masculine...) que celui des pilotes. Nous avons choisi de garder le seul groupe des pilotes, afin de ne pas alourdir la démonstration.

${ }^{8}$ Il ne sera pas fait mention ici des assignations de postes, dans le cadre de la division sociale du travail, liées à des critères de 'race', d’âge ou de santé.
} 


\section{Des dispositifs institutionnels favorables à l'initiative individuelle ?}

Deux dispositifs principaux, le Droit individuel à la formation (DIF) et la Validation des acquis de l'expérience (VAE), sont aujourd'hui à la disposition des salariés pour rendre possible la prise en charge de leurs parcours professionnels.

Le DIF est un dispositif ouvert par la récente loi de mai 2004 sur la formation professionnelle. Il permet à chaque salarié de demander l'accès à une formation de son choix, sur son temps personnel ou non. Ce n'est pas un droit en tant que tel, puisque l'octroi d'une telle formation reste du ressort de l'employeur. Ce droit est de vingt heures annuelles, soit la moyenne nationale, cumulables jusqu'à cent vingt heures sur six ans. Ce dispositif vise à étendre la formation professionnelle aux catégories qui en bénéficient le moins, par exemple les salariés des petites entreprises, les salariés les moins qualifiés, les femmes. Le rapport Péry (1999) note que 19,5 \% des femmes accèdent à la formation pour $21,6 \%$ des hommes. Cet écart augmente en cas de mariage (19\% des femmes pour $23,4 \%$ des hommes), de temps partiel (19,1\% des femmes pour $27,1 \%$ des hommes), du niveau de qualification (16,1\% des employées pour 26,2 \% des employés). La promotion de l'initiative individuelle (Lichtenberger, Méhaut 2001, par exemple) part du présupposé, qui reste à prouver, que ce sont les groupes socioprofessionnels les moins formés qui sont les plus demandeurs de formation. La loi - et les organisations syndicales et professionnelles qui avaient préparé cette évolution — visent donc, par ce type de dispositif, à ce que ces catégories aient accès à la formation.

La VAE permet aux salariés de valider leur expérience professionnelle, en vue d'obtenir un diplôme ou une certification reconnue. Le processus de certification s'opère par la validation, auprès d'une commission, d'un dossier présentant les phases successives de l'activité professionnelle et les compétences acquises. Sont aussi mises en évidence les compétences acquises dans le domaine social, associatif, syndical, mais aussi, pourquoi pas, dans le domaine familial. L'ensemble de la certification 
peut être obtenue sans formation complémentaire, contrairement au dispositif précédent (la Validation des acquis professionnels) qui reposait sur une formation complémentaire obligatoire. Le diplôme ainsi obtenu a, théoriquement, la même valeur que celui obtenu à l'issue d'une formation professionnelle.

Il a été souvent fait mention du bénéfice particulier que trouveraient les femmes à l'instauration de la reconnaissance de l'expérience par les nouveaux dispositifs instituant la VAE. Ce serait l'occasion, suivant Hélène Mignon (2000), de prendre en compte les compétences extérieures telles qu'associatives ou sociales, dans lesquelles les femmes s'investissent particulièrement. Même si cet investissement supérieur des femmes est contesté (Neyrat 2003), l'ouverture à la validation de compétences autres que professionnelles est revendiquée par certains auteurs car elle permet de compenser, pour les femmes, l'inégalité des situations.

Les femmes développent souvent des compétences dans des situations jusqu'à présent mal prises en compte [telles] les mères de famille interrompant leur carrière pour élever leurs enfants ou pour prendre en charge une personne proche gravement handicapée (Audige et al. 2005, p. 130).

Il faudrait alors " admettre l'expérience acquise dans un cadre familial pour certaines certifications d'aide à la personne » (id., p. 131). Mais c'est oublier que la sphère professionnelle ne reconnaît pas ces compétences acquises dans la sphère domestique. Celles-ci ont tendance à être naturalisées et renvoyées à des capacités ou des qualités propres aux femmes (Kergoat et al. 1992). Pour légitimer l'absence de prise en compte ou la dévalorisation de ces compétences, la structuration de la qualification et la division sexuelle du travail s'appuient sur des mécanismes mettant en jeu la représentation du travail et de sa valeur (Kergoat 2000). Ce premier processus symbolique s'accompagne d'une seconde étape aboutissant à dévaloriser le travail des femmes dans la sphère professionnelle. La légitimation de l'expérience est elle-même un produit de rapports sociaux qui octroient une valeur symbolique différente à l'expérience suivant la lecture sociale d'un métier. Toutes les activités ne se valent pas, tous les métiers ne sont pas considérés de la même manière, tous 'n'apportent' pas aux salariés en exercice une expérience 
‘équivalente'... Et cette lecture différenciée du travail ressort d'autant plus quand on l'observe à travers le prisme du genre (Barnier 2007).

\section{Métiers 'masculins' versus métiers 'féminins'}

L'étude de trois activités du transport aérien, l'enregistrement des passagers et le service en cabine d'une part, l'activité de pilotage de l'avion d'autre part, permet de comprendre le fonctionnement de ces dispositifs.

Les agents du passage enregistrent le passager se présentant au comptoir. Ils lui assignent un siège, le débarrassent de ses bagages, le libèrent des dernières contraintes terrestres pour lui permettre l'accès à bord. Ils sont chargés de la tâche ingrate de convaincre les passagers d'abandonner de plein gré leur liberté au nom à la fois de l'efficacité industrielle et de l'ordre aéronautique constitutif de la sécurité. C'est à l'agent de passage que revient de donner une autre forme sociale à ce citoyenpassager, lui retirant le droit à la parole, à la contestation, voire à l'initiative, dès qu'il est enregistré. Le traitement de milliers de passagers dans l'emplacement restreint de l'aérogare et dans le temps limité de l'embarquement renforce la nécessité de produire les conditions de sa passivité dans le cadre d'un ordre industriel. Ce rôle de sécurité et cette 'virtualisation' du passager dépassent donc largement la simple 'relation de service' que pourrait représenter à première vue l'enregistrement du passager.

L'hôtesse de l'air et le steward, aussi appelés 'personnels navigants commerciaux', sont deux figures mythiques du personnel des compagnies aériennes. Un certificat d’État reconnaît leur rôle en matière de sécurité et le niveau de leurs salaires témoigne d'une certaine reconnaissance sociale. Dans la cabine, règne le travail prescrit. Le vol est un ensemble de phases qui se succèdent, chacune minutée. Prenant le relais des agents de passage qui organisent l'embarquement, les personnels navigants commerciaux gèrent l'installation à bord et assistent les passagers qui se recréent un univers familier face à l'angoisse du vol. L'hôtesse de l'air accueille à bord les passagers comme une maîtresse de maison ses hôtes. Ce service complète la res- 
ponsabilité de ces personnels en matière de sécurité, s’appuyant sur une attention permanente envers chacun de leurs hôtes et une capacité à répondre aux situations d'urgence.

Le troisième métier retenu ici diffère totalement des deux premiers. Le groupe professionnel des pilotes - ou 'personnels navigants techniques' - a réussi à se faire reconnaître comme métier dès l'origine du transport aérien. Cette activité engage tout entier le corps et l'esprit du pilote. Celui-ci s'appuie sur l'avion lui-même, avec son appareillage technologique, qui lui permet de s'opposer autant à la nature envisagée comme une ennemie qu'à la panne du système lui-même. Les deux modèles de l'autorité et du paradigme technique s'affrontent depuis le début des années 1990. Dans l'ancien modèle, marqué par l'enracinement du transport aérien dans la seconde guerre mondiale, l'autorité du pilote s'affirmait à partir du risque assumé et surmonté dans le cadre hiérarchique de l'armée. L'entrée des stewards dans la cabine, sur le modèle de la marine, complétait dans la représentation du travail ce tableau de l'autorité sans partage du pilote - commandant de bateau. Dans le modèle actuel, le pilote est devenu celui qui garantit la sécurité. Le danger est toujours là, mais le risque est conjuré par la technique. Pour appuyer son autorité sur les autres groupes professionnels, il bénéficie de l'aura du commandant de bord, survivant de l'ancien modèle, ce que la compagnie aérienne ne se prive pas d'utiliser.

L'évolution professionnelle des salariés de ces trois groupes s'inscrit dans la dimension 'genrée' de l'activité du transport aérien. Les métiers du transport aérien se distinguent suivant deux ensembles caractérisés principalement par leur composition sexuée et leur représentation symbolique. Un regard porté préalablement sur le métier particulier des pilotes, conçu comme un idéal type du métier masculin, permet de dégager en creux les caractéristiques des autres métiers ici étudiés.

La caractéristique de métier masculin peut sans conteste être attribuée au métier de pilote, d'abord par la composition quasi masculine de ce métier (95,4\% d'hommes à Air France). À cette composition quasi unisexe s'ajoute la représentation traditionnelle de ce métier, qui s'inscrit dans une construction 'genrée' de l'activité. La capacité technique, la force nécessaire pour 
tenir le manche sur les anciens types d'avion, et surtout le pouvoir lié au rôle de commandant de bord relèvent en effet d'un registre symbolique typiquement masculin. Les modes de recrutement, soit par le canal de l'armée, soit par les classes préparatoires scientifiques (où les femmes ne représentent que 29,2 \% des effectifs), contribuent à rendre légitime cette prédominance. Comme cela a pu être montré ailleurs :

Le genre des organisations y est conçu comme le résultat d'une construction sociale et symbolique visant à faire du masculin (et, en creux, du féminin) le critère d'évaluation et d'appréciation des individus (Malochet 2008).

Cependant, la représentation traditionnelle est de plus en plus mise à mal par les évolutions, notamment technologiques : par exemple, le manche traditionnel est devenu une petite poignée qui se manie d'une main. Ces corps de métiers exclusivement ou quasi exclusivement masculins, comme ici les pilotes, relèvent de ces groupes professionnels où "l'identité masculine est au cour de la représentation identitaire du groupe " (Pfefferkorn 2006). Des mécanismes institutionnels très forts tels que l'interdiction d'accès, liée notamment historiquement à la nature militaire de ces emplois, ont longtemps empêché les femmes d'y entrer. Leur arrivée récente s'est en outre heurtée à "l'amitié virile du cockpit » (Mills 1998). Mais dans le même temps leur arrivée, même en nombre très limité, est aussi en phase avec une reconstruction de l'activité de commandant de bord autour de la figure nouvelle d'un 'commandant de bord animateur' à la tête d'une équipe technique et commerciale et maîtrisant le dialogue, nouvelle capacité désormais valorisée.

Les deux autres métiers étudiés ici, les personnels navigants commerciaux et les agents de passage, sont aux deux tiers composés de femmes. Nous proposons de leur attribuer le qualificatif de métier 'féminin' en raison de leurs caractéristiques qui sont opposées à celles du premier groupe. C'est ainsi par exemple que relève du registre féminin le traitement de la personne par les agents de passage, ou le service commercial à bord de l'avion. La mission du passage est décrite comme suit : 
Assurer le service aux passagers et aux personnes qui les accompagnent, lors des opérations d'enregistrement (Observatoire européen des métiers de l'aéronautique, FNAM 2000).

Si la première compétence relevée par cet observatoire renvoie à l'utilisation des systèmes informatiques, la seconde est d' « adapter sa présentation, son expression, son comportement aux exigences d'un accueil de qualité ». Dans la cabine, l'hôtesse de l'air doit jouer le rôle de l'hôtesse de maison, accueillant comme chez elle le passager afin de le rassurer. Sa présentation, son sourire sont autant des critères commerciaux que des éléments fondamentaux de la sécurité à bord. Constatant partout cette concentration des femmes sur certains métiers, Claude Grandin, Margaret Maruani et Hélène Yvonne Meynaud (1989) notaient déjà il y a vingt ans que :

Ce sont des métiers où le sourire, le charme, la gentillesse, la présentation, qualités classiquement considérées comme féminines, entrent parmi les critères de sélection, même si d'autres compétences, moins considérées comme spécifiquement féminines, sont également requises (bilinguisme).

Ces deux métiers s'effectuent presque constamment sous le regard des passagers. Cette 'représentation permanente' en constitue un autre élément féminin, contrainte spécifique s'appliquant plus particulièrement aux femmes, pour qui « il faut par-dessus tout rester “féminines”, c'est-à-dire séduisantes » (Duru-Bellat 1990, p.167). La relative mixité de ces métiers n'interdit donc pas de les qualifier de féminins, car à l'inverse des femmes, les hommes possèdent la «possibilité asymétrique de s'approprier attributs et rôles du sexe opposé » (Löwy 2006, p. 43).

Chacun de ces métiers s’inscrit dans des oppositions hiérarchiques qui structurent les rapports professionnels. La plus immédiate est la relation entre le commandant de bord et hôtesses de l'air. Ici le couple cockpit/cabine, deux zones dont l'étanchéité se renforce aujourd'hui avec les mesures sécuritaires, renvoie à celui du technique/commercial puisque le pilotage est d'abord envisagé comme une activité technique, comme le démontrent les multiples appareillages, et l'activité de cabine comme un service commercial où le rôle de sécurité ressort davantage 
d'attitudes et de réflexes que de savoirs directs. Cette division structurante dans l'avion rappelle celle tout aussi structurante entre les chirurgiens, majoritairement masculins, et les infirmières. Elle est homologue fondamentalement à la répartition sexuelle des rôles au sein du couple (Löwy 2006, p. 182). Ce renvoi des hôtesses de l'air au statut de femmes d'accueil est d'autant plus curieux que le métier de personnel naviguant commercial fut, à ses débuts en 1945, le résultat de l'arrivée conjointe de stewards, issus de la marine et amenant la tradition hôtelière, et d'infirmières de l'armée (IPSA) apportant à bord la technique de sécurité (Barnier 1999).

De même, le passage, activité à dominante féminine, où l'on traite le passager dans le cadre d'une relation humaine, contraste avec le pilotage où l'avion constitue l'objet du travail. Humain d'un côté, objet de l'autre, à nouveau cette opposition rejoint celle femmes/hommes. Dans la relation passager-agent d'enregistrement, les agents de passage, majoritairement des femmes, sont engagés dans une relation de service vis-à-vis d'un ensemble longtemps composé majoritairement d'hommes d'affaire et de cadres, la 'femme d'affaire' ou la 'femme cadre' représentant encore une figure largement minoritaire. La mise en relation de ces deux groupes aux caractéristiques fortement sexuées ne peut que reproduire le décalage de position entre hommes et femmes dans la société.

Les rapports particuliers au savoir s'expriment à travers ces dichotomies fondamentales qui traversent les relations professionnelles. Le modèle de l'hôtesse de l'air est souvent sollicité dans les publicités avec les qualités d'accueil, de gentillesse ou de disponibilité. A contrario, celui du pilote lui oppose l'autorité non partagée et la sécurité s'insérant dans un paradigme technique (Barnier, Rozenblatt 1997). Cet archétype du monde masculin apparaît comme une forme sociale dynamique, intégrant une évolution individuelle des agents dans un continuum de qualification. Ces métiers préservent des espaces de mobilité internes importants, au point que les individus y effectuent leur carrière sans rupture de vie professionnelle. C'est justement à ce modèle repéré que les 'métiers féminins' sont confrontés. Danièle Kergoat (1992) avait noté, à propos des infirmières, cette relation différente entre savoir et expérience, mettant en 
évidence la nécessité pour les infirmières de différencier la personne et le métier exercé et de construire le relationnel comme une qualification. De même, en défendant la dimension de métier de leur activité, les personnels navigants commerciaux et les agents de passage affirment la légitimité des savoirs professionnels, relationnels, techniques et de sécurité, acquis dans le cadre d'une expérience professionnelle cumulative.

\section{Manque de reconnaissance et mal-être, deux moteurs de l'initiative}

Dans les deux métiers, du passage et de la cabine, la reconnaissance de l'expérience professionnelle reste limitée et pousse certains agents à entrer dans la logique de parcours. On peut faire une observation analogue en ce qui concerne l'effet du mal-être au travail.

Chez les personnels navigants commerciaux, le service commercial et la technicité de sécurité sont perçus par les responsables d'entreprise comme reposant tous deux sur des tâches liées à des compétences de comportement. La dimension de sécurité n'est pas prise en compte. Celle-ci repose sur une empathie avec le passager et donc une grande capacité d'adaptation culturelle à tous les publics, ainsi que la maîtrise de plusieurs langues et la connaissance des gestes techniques de secourisme et des automatismes de sécurité. Ces compétences ne peuvent que s'accroître dans la pratique continue de ce métier, assurant ainsi toujours davantage de sécurité dans la cabine. La promotion comme chef de cabine, puis chef de cabine principal implique cependant la réussite à un concours, et sa validation par la hiérarchie lors de l'occupation d'un poste laissé vacant. Ici, l'expérience professionnelle est validée par la promotion au sein de la filière professionnelle, validation inégalitaire ${ }^{9}$ suivant le sexe malgré une activité identique.

\footnotetext{
${ }^{9}$ La structure hiérarchique de ce métier se traduit par $68 \%$ de femmes au niveau hôtesse et steward, $61 \%$ au niveau chef de cabine et $45 \%$ au niveau chef de cabine principal, pour culminer à trois femmes pour onze hommes au plus haut niveau des personnels navigants commerciaux (Bilan social Air France 2003).
} 
Les 'compétences', qui recouvrent ici davantage les dimensions commerciales du métier, ne sont pas reconnues. Pourtant, la dimension de technique relationnelle incluse dans cette activité est réelle, que ce soit dans la gestion des conflits ou dans la connaissance du fonctionnement de l'aéroport, de l'arrivée ou de l'enregistrement.

J'ai eu chez Aéroports de Paris une formation plus ou moins psychologique sur l'agression, la gestion des éléments internes et cela m'a aidée dans certains moments où je me suis fait agresser verbalement (une employée d'enregistrement).

Mais la formation, notamment à Air France, prend davantage l'aspect de l'intégration dans un 'moule' :

Plutôt qu'apprendre aux gens à bien se coiffer, bien se manucurer, bien se maquiller, on ferait bien de leur apprendre les bases du métier et leur mettre en main les atouts pour ne pas se faire bouffer (une femme personnel navigant commercial).

Se trouve alors en question l'absence de formalisation d'un savoir des activités de service, non repérable et donc non cumulable, alors même que c'est l'ancienneté et l'expérience qui, de l'avis de tous, permettent de gérer, par exemple, un passager indiscipliné ou une foule en colère face au comptoir lorsque l'avion est fortement retardé. Le manque de reconnaissance dans le métier amène les agents à envisager la nécessité du changement d'emploi pour assurer leur évolution professionnelle. La perspective de la mutation est un des moteurs de l'engagement des salariés dans leur travail :

Le seul objectif, c'est de sortir du passage (Flottes, Rebeyrat 2001).

Le souhait porte majoritairement les agents de passage vers les tâches de trafic, telles que l'organisation des vols et des plannings, de formation ou toute autre activité. Mais ce sont des emplois reposant sur des dimensions techniques, aspects non valorisés dans les emplois du passage.

La comparaison avec le groupe professionnel des pilotes met en évidence les caractéristiques particulières attribuées au travail commercial. Chez les pilotes, l'expérience professionnelle, conçue dans une continuité de travail, est au centre de la valorisation du travail. Son accroissement accompagne l'élargissement 
des responsabilités (liées à la dimension croissante des avions pilotés au cours de la carrière) et la progression des salaires. Mais toute l'approche du travail donne un rôle premier à la connaissance théorique. C'est ainsi que le groupe des pilotes représente la quintessence de la domination d'un savoir technique aéronautique, partagé avec d'autres groupes professionnels tel que celui des mécaniciens avions. Ce paradigme technique, au sein duquel se différencie la valeur distribuée à chaque métier, donne à la grille de classification une légitimité admise de tous. La technique contribue ici à "distribuer les formes d'intelligence socialement acceptables » (Vincent 1995). Avec les mécaniciens avions, les pilotes assoient leur pouvoir sur "l'appropriation de la machine" (Cockburn 2004). Connaissance et pouvoir se conjuguent pour rendre légitime le rôle prépondérant donné à ces métiers dans les grilles de classification. De cette première lecture ressort un apprentissage centré sur les savoirs techniques, et appuyé, dans le premier groupe des pilotes, sur des diplômes techniques ; sur l'expérience de communication qui s'acquiert dans la pratique, dans le second groupe, celui des agents de passage et personnels navigants commerciaux.

La valorisation du premier métier, la dévalorisation des autres trouvent ainsi des justifications dans le monde symbolique de la représentation sociale du travail. La structuration du savoir professionnel se réfère alors à deux modèles types opposés : d'un côté le pilote, porteur du paradigme technique, inscrivant le travail au sein d'une progression dans un corpus technique, et reposant sur une légitimité sociale; de l'autre, l'agent de passage, titulaire d'un savoir morcelé, non cumulatif, dont la participation à l'effort général de sécurité repose sur des comportements plutôt que sur des savoirs normés, et dont l'accès à la technique (gestion des vols, prise en main des systèmes de réservation informatiques) n'est pas reconnu. La relation au savoir professionnel est ainsi largement tributaire des rôles sociaux impartis aux groupes professionnels. Cette dévalorisation est intégrée au fonctionnement de l'entreprise à travers la division du travail. La 'naturalisation' des différences entre hommes et femmes à travers ces processus symboliques conduit à les masquer : 
Le processus de ségrégation qui prend des formes multiples n'est pas perçu comme tel par les femmes (Degenne 2004).

La dévalorisation du travail s’accompagne d'une impossibilité de valoriser l’expérience dans le même métier. Or, comme le note Paul Santelmann (2007), l'un des moteurs de la formation est la perspective de pouvoir en tirer un avantage en termes de mobilité ascendante. C'est donc par le changement d'activité que les salariés du passage et du service à bord envisagent leur évolution professionnelle.

Ces ruptures s’imposent d'autant plus que ces activités sont vécues comme engendrant des conditions de travail pénibles, comme le mettent en avant, lors des entretiens, les agents rencontrés. Les personnels de cabine se voient en effet soumis à des variations de pression permanentes, des vibrations continues, des chocs répétés qui se traduisent par de fréquentes lombalgies. La pénibilité provient aussi, pour les salariés du passage comme pour les PNC, de la tension que crée la gestion de passagers par ailleurs de plus en plus stressés, dans un contexte de réduction des effectifs qui limite les marges de manœuvre des personnels (Bertolini et al. 2003). Ajoutons enfin l'importance des horaires dans cette activité de transport, où les deux tiers des agents travaillent en horaires décalés. Ces caractéristiques usent les organismes. Le conflit des personnels navigants commerciaux d'Air France (grève suivie à 83 \%, pendant quatre jours, début novembre 2007) a mis sur le devant de la scène cette pénibilité ressentie si fortement. L’enquête effectuée pour Air France par un cabinet d'audit, Altedia (février 2008), met parmi les premières causes de mécontentement le planning et la charge de travail. Les rotations et la fatigue liée au service à bord sont les éléments qui reviennent le plus souvent dans les motifs du conflit. Une autre étude sur la pénibilité du travail des personnels navigants commerciaux sur des vols moyen-courrier (Rapport médical au CHS-CT, 2006) montre ainsi que seulement $20 \%$ des personnel navigants commerciaux ont eu plus de six heures de sommeil la veille du départ pour des rotations commençant très tôt. Ces vols qui engagent les personnels sur des tronçons successifs (Paris - Madrid - Paris - Amsterdam - Paris par exemple) se révèlent très fatigants. 
La surqualification des salariés engendre aussi insatisfaction et désir de changement. Pour les agents du passage et les personnels navigants commerciaux, le décalage est important entre les niveaux de formation réels et le niveau validé : bien que relevant de métiers reconnus tous deux comme équivalents à des emplois qualifiés de niveau Bac, la population qui les compose est largement plus qualifiée (90\% des personnels navigants commerciaux ont le niveau $\mathrm{Bac}+2$ et $60 \%$ ont le niveau Bac +4 , de même $60 \%$ des agents de passage ont le niveau Bac +2 ). Ceci n'est certes pas spécifique à ces emplois, même si le décalage est plus important que pour d'autres métiers.

La pénibilité des métiers et l'insatisfaction au travail amènent les salariés à s'engager dans des processus de progression dans le parcours professionnel. Cependant, en l'absence de progression possible des salariés dans leur domaine professionnel (passage, cabine), le désir d'évolution se déplace vers la recherche d'une progression ailleurs, dans d'autres segments professionnels. L'engagement dans de tels processus relève donc beaucoup plus de la soupape de sécurité vis-à-vis de situations intenables, que de la recherche d'une véritable valorisation professionnelle. Cette aspiration à une progression de carrière conduit à la rupture du parcours professionnel.

\section{Collectifs stables et contrainte gestionnaire, obstacles à l'initiative individuelle}

Cette forme de relation particulière, laissant une place marginale à la construction du parcours au sein d'un métier, entre par ailleurs en résonance avec les modes de gestion d'une grande entreprise et la nécessité de constituer des collectifs de travail stables.

Le métier permet de gérer les aléas, chaque professionnel faisant confiance à ses collègues. L'hôtesse ou le steward, dans la cabine, qui voit son collègue en difficulté face à un passager excité et non obéissant, vient lui prêter main forte. Derrière sa banque d'enregistrement, l'agent de passage garde un œil sur son collègue, aux prises avec un dossier informatique difficile. 
Le pilote et le copilote s'épaulent et se surveillent mutuellement (principe du crooscheck), et doivent pouvoir se signaler leur faute au-delà de toute barrière hiérarchique. Le métier représente ici " l'institutionnalisation d'une communauté de culture et d'action » (Segrestin 1975, p. 169) permettant la constitution d'une communauté professionnelle. L'appartenance reconnue au collectif de métier accompagne l'attribution d'une compétence équivalente à la sienne. Mais équivalence ne veut pas dire similitude. Chacun a sa propre expérience, untel connaîtra mieux le vin à servir dans la cabine, tel autre aura déjà effectué un accouchement en plein vol et connaîtra les gestes essentiels dans ce cas (heureusement peu fréquent). Le collectif de travail s'envisage ici comme une entité plurielle, mouvante et se reconstituant durant chaque vol à partir d'un ethos partagé, collectif où les parcours, origines, diplômes, qualifications diverses se complètent et s'enrichissent.

La stabilité caractérise ces collectifs de métier. Dans les deux métiers étudiés, qui ne relèvent pas du domaine technique et ne s'inscrivent pas dans un corpus de connaissances aéronautiques comme pour les mécaniciens avions (Barnier 2000) et les pilotes, les savoirs ressortissent davantage d'un savoir-être devant les passagers, d'un savoir-faire dans le traitement de la relation avec le passager ou d'un savoir expert, par exemple en matière de sécurité aérienne, savoir souvent non reconnu (Barnier 1999). La connaissance aéronautique, dans ce cadre non formel, intègre une familiarité avec l'environnement, l'avion, l'aérogare, tous ces lieux dont la possession relève de chaque métier : aux agents d'enregistrement l'aérogare, aux personnels navigants commerciaux la cabine. La formation commerciale ou en langues représente la majeure partie des formations non réglementaires. La formation professionnelle est importante (en moyenne, de quatre jours par an environ pour les métiers du passage, sept jours chez les personnels navigants commerciaux), mais elle est surtout de type réglementaire (65\% des formations pour le personnel navigant commercial, $33 \%$ pour le personnel au sol en moyenne dans la compagnie aérienne étudiée), ce qui oblige à asseoir cette connaissance spécifique sur l'apprentissage dans le cadre de l'activité quotidienne et donc sur la stabilité du collectif. 
Pour les métiers du passage et de la cabine, les restructurations actuelles à Air France, suite à la privatisation (2004) et aux restrictions d'embauches, ne sont pas favorables à des mutations. Le récent accord triennal ${ }^{10}$ sur la gestion des emplois (2006-2009) promeut pourtant une mobilité fondée sur la reconnaissance des ressources internes. Il précise que :

Au service de cette mobilité, les dispositifs de formation sont mobilisés en cohérence avec l'accord de branche (p. 6).

Mais l'initiative individuelle en matière de formation n'est pas considérée comme un élément de gestion du personnel. Sur l'ensemble de la principale compagnie aérienne considérée, ce sont 300 stages de formation correspondant à 6000 heures en 2005 qui s'effectuent dans le cadre du DIF pour 45000 personnels au sol, et 110 formations pour les 15000 personnels navigants commerciaux; et si les chiffres sont multipliés par trois pour l'année suivante, cela reste marginal dans cette compagnie, du moins dans les premières années de ce dispositif. Les formations se dirigent principalement vers la bureautique et les langues, un laboratoire de langue étant mis à la disposition des personnels. Le mode de propagande pour cette nouvelle mesure explique certainement, en partie, cette réserve : la diffusion des informations concernant cette nouvelle opportunité a suivi principalement le canal hiérarchique. Le moment privilégié de discussion sur un projet de formation individuel est désigné comme celui de l'évaluation annuelle avec le supérieur hiérarchique. La compagnie explique que l'effort important de formation dont bénéficie le personnel ( $8,7 \%$ de la masse salariale en 2006) ne peut être augmenté actuellement, et renvoie donc l'usage du DIF à un choix personnel du salarié.

L'accès au DIF reste donc largement entre les mains de la hiérarchie. Cet accès se fait de plus principalement sur le temps individuel, notamment pour les PNC. Le rapport Péry posait déjà, en 1999, cette question :

Comment faire coexister plusieurs principes qui doivent régir notre système: celui d'une obligation faite aux entreprises d'adapter leur main-d'œuvre aux mutations technologiques et organisationnelles, celui de la recherche d'une plus grande

${ }^{10}$ Accord signé par les syndicats FO, CFDT, CGC et UNSA. 
convergence d'intérêts entre l'employeur et le salarié en faveur du développement des compétences et de l'autonomie professionnelle et celui de la liberté donnée à chacun de suivre la formation de son choix dans le cadre d'éducation permanente? (1999, p. 45).

Si la formation d'adaptation est bien effectuée par l'entreprise, la liberté de choisir une formation, pour le salarié, est limitée en l'absence d'une possibilité d'évolution professionnelle. L'initiative se révèle "contrainte » et peut rester une «vue de l'esprit » (Maggi-Germain 2004) si le salarié n’en maîtrise pas les finalités.

L'initiative n'existe pas à l'état pur, elle est l'expression de tensions entre contraintes et autonomie (Correia 2004).

Les projets des salariés ne peuvent se réaliser que si l'individu est capable de confronter ses envies aux possibilités ouvertes par l'organisation où il se trouve. Mais justement, ce sont les catégories de population où se retrouvent les plus défavorisés en matière de formation (id.).

En ce sens, le DIF, s'appuyant sur la mythification d'une 'initiative individuelle' abstraite, ne peut que renforcer les inégalités d’accès à la formation.

\section{Une relation imaginaire}

Dans le transport aérien, l’organisation du travail est fondée sur la stabilité du collectif de travail et l'importance accordée au métier. Mais, contrairement au groupe professionnel des pilotes, pour les deux métiers 'commerciaux' 'féminins' considérés, la faible formation professionnelle renforce la dimension informelle de l'apprentissage du métier. Dans le même temps, les aspirations à l'évolution ne peuvent se concevoir que dans la rupture vis-à-vis du groupe professionnel. La stabilité revendiquée du groupe professionnel et l'assimilation de tout départ à une rupture, vécue sur un mode individuel, traduisent une résistance des collectifs de travail. Trois éléments peuvent être avancés pour interpréter l'imperméabilité de l’organisation du transport aérien à l'initiative individuelle dans ces métiers dits ‘féminins'. 
C'est d'abord le cadre contraint du travail qui doit être mis en avant. L'organisation du travail repose sur le principe d'autorité qui puise ses sources dans les configurations professionnelles d'origine du transport aérien. Calqué sur l'organisation militaire du travail (les pilotes de la compagnie aérienne anglaise devaient être pilotes de la RAF jusque dans les années 1980, ce qui excluait les femmes) (Mills 1998), le modèle masculin de l'autorité du pilote, seul maître à bord, se diffuse dans la compagnie pour imposer un rapport hiérarchique fondé sur l'obéissance. Venant compléter ce rapport hiérarchique, la normalisation du travail se trouve en progression dans l'ensemble des activités. La responsabilisation des agents dans le travail quotidien vient compenser ce modèle initial rigide et lui fournir l'ingrédient indispensable de l'implication humaine dans le travail. Il faut noter ici la contradiction existant entre cet engagement de nature de plus en plus individuelle dans ce secteur aéronautique, et l'absence de prise en compte de l'initiative individuelle que nous avons notée et qui tend à déresponsabiliser le salarié.

Le deuxième élément est lié à une gestion des emplois qui n'admet pas le changement à partir de l'initiative individuelle. L'usage d'une liberté de décision quant à son propre avenir ressort du rapport au pouvoir, question qui occupe une place prépondérante pour les femmes dans l'entreprise. La reconnaissance de l'expérience à travers la seule progression par poste, pour les métiers 'féminins' que nous avons étudiés, dépend ici du pouvoir hiérarchique. Cette question du pouvoir est au centre des rapports sociaux. Les deux principes mis en évidence par Danièle Kergoat (2000) à propos de la division sexuelle du travail, le principe de division et le principe de hiérarchisation entre les tâches féminines et masculines, se rencontrent ici. L'organisation du travail apparaît liée à la structuration du pouvoir. La structuration de l'expérience, quant à elle, est liée au regard porté sur le travail sexué. Et justement :

La 'richesse' du travail dépend largement de la position hiérarchique utilisée (Neyrat 2003, p. 233).

Fondamentalement, c'est le droit à l'initiative des femmes, dans le cadre professionnel comme ailleurs, qui se trouve posé. 
Enfin, troisième élément de réponse, la division sociale du travail, au centre de la relation au métier dans la configuration du transport aérien, se double d'une division sexuelle du travail. En naturalisant les fonctions remplies par les agents dans le service au passager, au sol ou en vol, celle-ci rend légitime l'assignation des agents (femmes) à leur activité. Or c'est justement à l'encontre de cette répartition des tâches que viendrait se heurter l'initiative individuelle en matière de formation et de parcours professionnel. La présomption d'un individu (et notamment des femmes, pourtant plus demandeuses des congés individuels de formation [Péry 1999, p. 143]) de sortir du rôle qui lui est assigné de par son identité sociale, ne peut qu'être ramenée à de plus justes proportions : chacun·e à sa place, tel est la consigne aéronautique.

L'appel à l'initiative individuelle relève alors de la construction d'une relation imaginaire entre le salarié et son emploi. Les outils tels que la VAE ou le DIF ne sont donc pas « des instruments de pilotage des parcours professionnels déconnectés du lien de subordination entre employeur et salarié » comme l'indique Sylvie Monchatre (2007), mais plutôt des dispositifs s'inscrivant et prenant sens dans des contextes sociaux précis. Libre fictivement de réaliser son projet, il/elle se retrouve d'autant plus lié·e à sa situation présente qu'il/elle est censé·e en être responsable. Le parcours exprime dans cette vision l'individualité au sein du collectif, et le collectif de travail devient lui-même un croisement de parcours individuels. Un glissement s'opère alors de la responsabilité du salarié vis-à-vis de son adaptation au poste de travail, à sa responsabilisation vis-à-vis de son parcours.

\section{Références}

Audige Thomas, Debeaupuis Jean, Segal Patrick et al. (2005). Validation des acquis de l'expérience, du droit individuel à l'atout collectif. Paris, Inspection générale des affaires sociales.

Barnier Louis-Marie (1999). « Personnel navigant commercial, d'un savoir intuitif à un savoir d'expertise ». Formation emploi, $\mathrm{n}^{\circ} 2$, numéro spécial "Activités de travail et dynamique des compétences ». 
- (2000). «Le mécanicien avion, quand la logique compétence affronte le métier ». In Rozenblatt Patrick (ed). Le mirage de la compétence. Paris, Syllepse.

- (2005). Service public ou service privé ? La valorisation du travail dans le transport aérien. Thèse de doctorat, Université Paris $10-$ Nanterre, disponible sur : http://lmbarnier.free.fr.

- (2007). «L'expérience professionnelle au prisme du rapport de genre ». Cahiers de la recherche sur l'éducation et les savoirs, $n^{\circ} 6$.

Barnier Louis-Marie, Rozenblatt Patrick (1997). Ceux qui croyaient au ciel, enjeux et conflits à Air France. Paris, Syllepse.

Bertolini Renzo, Drewczynski Andrew, Rosskam Ellen (2003). Service on the Ground: Occupational Health of Airport Check-In Workers. Geneva, International Labour Organization.

Clavel Edith, Baunay Yves (eds) (2002). Toute la vie pour apprendre. Paris, Syllepse « Nouveaux regards ».

Cockburn Cynthia (2004). « Le matériel dans le pouvoir masculin ». Cahiers du genre, $\mathrm{n}^{\circ} 36$ «Les résistances des hommes au changement » (Anne-Marie Devreux, ed).

Correia Mario (2004). «Formation : de nouveaux droits. Quelle place à l'initiative individuelle ? » Paris, DARES, Actes du colloque Les évolutions de la formation professionnelle continue : l'impact de la loi du 4 mai 2004.

Degenne Alain (2004). «Avant propos ». In Genre et données longitudinales. $X I^{e}$ journées d'analyse longitudinale du travail. Marseille, CEREQ « Reliefs·4. Échanges du CEREQ ».

Duru-Bellat Marie (1990). L'école des filles : quelle formation pour quels rôles sociaux? Paris, l'Harmattan "Bibliothèque de l'éducation ».

Flottes Anne, Rebeyrat Jean-François (2001). Enquête de psychodynamique du travail : des agents du passage long-courrier (A \& C). Expertise du CHS-CT, $n^{\circ} 2$, Air France CDG.

Grandin Claude, Maruani Margaret, Meynaud Helène Yvonne (1989). « L’inégalité professionnelle dans les entreprises publiques à statut réglementaire ». Cahiers du GIP-Mutations industrielles, $\mathrm{n}^{\circ} 34$.

Hughes Everett C. (1937). "Institutional Office and the Person”. The American Journal of Sociology, vol. 43, $\mathrm{n}^{\circ} 3$.

Kergoat Danièle (2000). «Division sexuelle du travail et rapports sociaux de sexe ». In Hirata Helena et al. (eds). Dictionnaire critique du féminisme. Paris, PUF « Politique d'aujourd'hui » $\left[2^{\mathrm{e}}\right.$ éd. 2004]. 
Kergoat Danièle et al. (1992). Les infirmières et leur coordination, 1988-1989. Paris, Lamarre.

Lichtenberger Yves, Méhaut Philippe (2001). «Les enjeux d'une refonte de la formation professionnelle continue, bilan pour un futur ». Mimeo, $37 \mathrm{p}$.

Löwy Ilana (2006). L'emprise du genre: masculinité, féminité, inégalité. Paris, La Dispute « Le genre du monde ».

Maggi-Germain Nicole (2004). «La formation professionnelle continue entre individualisation et personnalisation des droits des salariés ». Droit social, ${ }^{\circ}$ 629, mai.

Malochet Guillaume (2008). «Surveillantes surveillées : la mixité au travail dans les prisons pour hommes ». In Guichard-Claudic Yvonne, Kergoat Danièle, Vilbrod Alain (eds). L'inversion du genre : quand les métiers masculins se conjuguent au féminin... et réciproquement. Rennes, Presses universitaires de Rennes.

Merllié Dominique, Prévot Jean (1991). La mobilité sociale. La Découverte « Repères ».

Mignon Hélène (2000). Rapport d'information fait au nom de la Délégation aux droits des femmes. Paris, Assemblée nationale, 13 décembre.

Mills Albert J. (1998). “Cockpits, Hangars, Boys and Galleys: Corporate Masculinities and the Development of British Airways”. Gender, Work \& Organisation, vol. 5, n 3, July.

Monchatre Sylvie (2007). « Des carrières aux parcours... en passant par la compétence ». Sociologie du travail, vol. 49, n 4.

Neyrat Frédéric (2003). « De l'éducation permanente à la certification permanente. La validation des acquis de l'expérience : levier de transformation de l'enseignement supérieur ». Cahiers de recherche sur l'éducation et les savoirs, $n^{\circ} 2$.

Péry Nicole (1999). La formation professionnelle, diagnostics, défis et enjeux. Secrétariat d'État aux droits des femmes et à la formation professionnelle.

Pfefferkorn Roland (2006). «Des femmes chez les sapeurspompiers ». Cahiers du genre, $\mathrm{n}^{\circ} 40$ « Hors-champ ».

Santelmann Paul (2007). «Enjeux syndicaux. L'enlisement de la formation professionnelle continue ». Revue de l'IRES, n 53.

Segrestin Denis (1975). « Du syndicalisme de métier au syndicalisme de classe : pour une sociologie de la CGT ». Sociologie du travail, vol. $17, \mathrm{n}^{\circ} 2$. 